\title{
Using the longitudinal space charge instability for generation of vacuum ultraviolet and $x$-ray radiation
}

\author{
E. A. Schneidmiller and M. V. Yurkov \\ Deutsches Elektronen-Synchrotron (DESY), Notkestrasse 85, D-22607 Hamburg, Germany
}

(Received 1 April 2010; published 13 November 2010)

\begin{abstract}
Longitudinal space charge (LSC) driven microbunching instability in electron beam formation systems of x-ray free-electron lasers (FELs) is a recently discovered effect hampering beam instrumentation and FEL operation. The instability was observed in different facilities in infrared and visible wavelength ranges. In this paper we propose to use such an instability for generation of vacuum ultraviolet (VUV) and $\mathrm{X}$-ray radiation. A typical longitudinal space charge amplifier (LSCA) consists of few amplification cascades (drift space plus chicane) with a short undulator behind the last cascade. If the amplifier starts up from the shot noise, the amplified density modulation has a wide band, on the order of unity. The bandwidth of the radiation within the central cone is given by an inverse number of undulator periods. A wavelength compression could be an attractive option for LSCA since the process is broadband, and a high compression stability is not required. LSCA can be used as a cheap addition to the existing or planned short-wavelength FELs. In particular, it can produce the second color for a pump-probe experiment. It is also possible to generate attosecond pulses in the VUV and x-ray regimes. Some user experiments can profit from a relatively large bandwidth of the radiation, and this is easy to obtain in the LSCA scheme. Finally, since the amplification mechanism is broadband and robust, LSCA can be an interesting alternative to the self-amplified spontaneous emission free-electron laser (SASE FEL) in the case of using laser-plasma accelerators as drivers of light sources.
\end{abstract}

DOI: 10.1103/PhysRevSTAB.13.110701

PACS numbers: 41.60.Cr

\section{INTRODUCTION}

Longitudinal space charge (LSC) driven microbunching instability $[1,2]$ in electron linacs with bunch compressors [used as drivers of short-wavelength free-electron lasers (FELs)] was a subject of intense theoretical and experimental studies during the past years [3-9]. Such instability develops in infrared and visible wavelength ranges and can hamper electron beam diagnostics and FEL operation.

In this paper we propose to use this effect for generation of vacuum ultraviolet (VUV) and x-ray radiation. We introduce a concept of an LSC amplifier (LSCA) and present basic scaling relations in Sec. II. We consider possible applications of such an amplifier in Sec. III, and end up with discussion and conclusions in Sec. IV.

\section{GENERIC LSC AMPLIFIER}

\section{A. Scheme of an LSCA}

Let us consider a scheme presented at Fig. 1. An amplification cascade consists of a focusing channel and a dispersive element (usually a chicane) with an optimized momentum compaction $R_{56}$. In a channel the energy modulations are accumulated, that are proportional to density modulations and space charge impedance of the drift space. In the chicane these energy modulations are converted into induced density modulations that are much larger than the initial ones [1]; the ratio defines a gain per cascade. In this paper we will mainly consider the case when the amplification starts up from the shot noise in the electron beam-although, in principle, the coherent density modulations can be amplified in the same way. A number of cascades is defined by the condition that the total gain, given by the product of partial gains in each cascade, is sufficient for saturation (density modulation on the order of unity) after the start up from shot noise. As we will see, in most cases two to four cascades would be sufficient. The amplified density modulation has a large relative bandwidth, typically in the range 50\%-100\%. Behind the last cascade a radiator undulator is installed, which produces a powerful radiation with a relatively narrow line (inverse number of periods) within the central cone. This radiation is transversely coherent, and the longitudinal coherence length is given by the product of the number of undulator periods by the radiation wavelength. When LSCA saturates in the last cascade, a typical enhancement of the radiation intensity over that of spontaneous emission is given by a number of electrons per wavelength.

\section{B. Formula for a gain per cascade}

Let us now present simple formulas for calculations of the gain and optimization of parameters of an LSCA. As in the case of a SASE FEL [10], we assume that at the entrance to the amplifier there is only shot noise in the electron beam. Let us consider the linear amplification of spectral components of the noise within the amplifier band. 


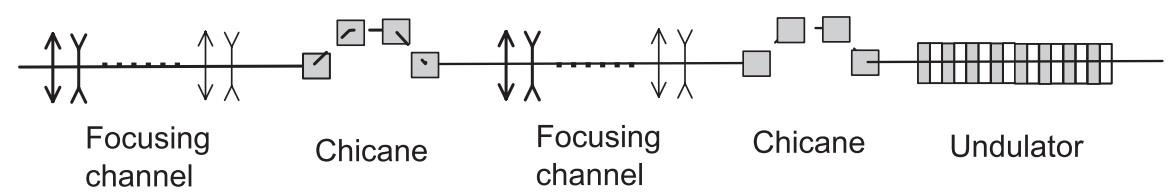

FIG. 1. Conceptual scheme of an LSC amplifier.

The formula for amplitude gain per cascade was obtained in studies of microbunching instabilities in linacs with bunch compressors [1]:

$$
G_{n}=C k\left|R_{56}\right| \frac{I}{\gamma I_{\mathrm{A}}} \frac{4 \pi|Z(k)| L_{d}}{Z_{0}} \exp \left(-\frac{1}{2} C^{2} k^{2} R_{56}^{2} \frac{\sigma_{\gamma}^{2}}{\gamma^{2}}\right) .
$$

Here $k=1 / \lambda=2 \pi / \lambda$ is the modulation wave number, $Z$ is the impedance of a drift space (per unit length), $Z_{0}$ is the free-space impedance, $L_{d}$ is the length of the drift space, $I$ is the beam current, $I_{\mathrm{A}}$ is the Alfvén current, $R_{56}$ is the compaction factor of a chicane, $C$ is the compression factor, ${ }^{1} \gamma$ is relativistic factor, and $\sigma_{\gamma}$ is rms uncorrelated energy spread (in units of rest energy). It is assumed here that energy modulations are accumulated upstream of the chicane but not inside: there the self-interaction is suppressed due to $R_{51}$ and $R_{52}$ effects [11-13]. We also assume in the following that a length of the drift space is much larger than that of the chicane, while the $R_{56}$ of the drift space is much smaller. Also note that the formula (1) was obtained under the condition of high gain, $G \gg 1$. In this case both the sign of $R_{56}$ and the phase of impedance are not important. ${ }^{2}$ The value of the $R_{56}$ is to be optimized for a highest gain at a desired wavelength.

In this paper we will consider LSC induced energy modulations in a drift space or in an undulator (at a wavelength that is much longer than the resonant one). In the latter case ${ }^{3}$ the relativistic factor $\gamma$ in the formulas for impedance should be substituted by the longitudinal relativistic factor $\gamma_{z}[14]$ :

$$
\begin{aligned}
\frac{4 \pi Z(k)}{Z_{0}}= & \frac{2 i k}{\gamma_{z}^{2}} \int d \vec{r}_{\perp}^{\prime} \\
& \times \int d \vec{r}_{\perp}^{\prime \prime} \rho\left(\vec{r}_{\perp}^{\prime}\right) \rho\left(\vec{r}_{\perp}^{\prime \prime}\right) K_{0}\left(\frac{k\left|\vec{r}_{\perp}^{\prime}-\vec{r}_{\perp}^{\prime \prime}\right|}{\gamma_{z}}\right) .
\end{aligned}
$$

Here $\rho\left(\vec{r}_{\perp}\right)$ is the transverse distribution of the current density and $K_{0}$ is the modified Bessel function. In particular, in the case of a pencil beam limit for the space charge,

$$
\lambda \gamma_{z} \gg \sigma_{\perp}
$$

\footnotetext{
${ }^{1}$ The wave number after the chicane is $C k$.

${ }^{2}$ If the gain is not high, the sign of $R_{56}$ is very important: for example, a dogleg would have a big advantage over a chicane.

${ }^{3}$ Under the condition $\sigma_{\perp}^{2} \gg \lambda \lambda_{w}$, where $\lambda_{w}=\lambda_{w} / 2 \pi$ is the reduced undulator period and $\sigma_{\perp}$ is rms transverse size of the beam.
}

$\sigma_{\perp}$ being an rms transverse size of the beam, the LSC impedance is given by

$$
\frac{4 \pi Z(k)}{Z_{0}}=\frac{i k}{\gamma_{z}^{2}}\left(2 \ln \frac{\gamma_{z}}{k \sigma_{\perp}}+\mathrm{const}\right) .
$$

Here the constant in the brackets depends on transverse shape of the beam.

We request that a density modulation does not change significantly in the drift space. This may happen due to plasma oscillations or due to a spread of longitudinal velocities for a finite beam emittance. Thus, a drift space length is limited by the condition

$$
L_{d} \leq \min \left(L_{1}, L_{2}\right) .
$$

Here $L_{1}$ is the reduced wavelength $\lambda_{p}$ of plasma oscillations:

$$
L_{1} \simeq \lambda_{p}=\gamma_{z}\left(\frac{I}{\gamma I_{\mathrm{A}}} \frac{4 \pi|Z| k}{Z_{0}}\right)^{-1 / 2} .
$$

The second limitation follows from the condition that the longitudinal velocity spread due to a finite beam emittance does not spoil the modulations during the passage of the drift:

$$
L_{2} \simeq \frac{\lambda}{\sigma_{\theta}^{2}}=\frac{\beta \lambda}{\epsilon},
$$

where $\sigma_{\theta}$ is the angular spread in the beam, $\beta$ is the beta function, $\epsilon=\epsilon_{n} / \gamma$ is beam emittance, and $\epsilon_{n}$ is the normalized emittance.

\section{Formulas for an optimized LSC amplifier}

Let us first consider the case without wavelength compression, $C=1$. We start optimization assuming that the beam parameters are fixed: current $I$, normalized emittance $\epsilon_{n}$, beam energy $\gamma$ and energy spread $\sigma_{\gamma}$ in units of the rest energy, and longitudinal gamma-factor $\gamma_{z}$. We can select a central wavelength, optimize $R_{56}$ of the dispersion section for the chosen wavelength, choose beta function, and optimize a length of the drift space. Our goal is to get a highest gain at a shortest wavelength.

The impedance (2) increases with $k$, achieves the maximum at

$$
\lambda \simeq \lambda_{\mathrm{opt}} \simeq \frac{\sigma_{\perp}}{\gamma_{z}}=\frac{\sqrt{\epsilon \beta}}{\gamma_{z}},
$$

and then decays in the asymptote of a pancake beam. Let us consider the wavelength about $2 \pi \lambda_{\text {opt }}$ as an optimum 
choice, since the impedance is the largest, and transverse correlations of the LSC field are still on the order of the beam size [4]. The impedance at this wavelength can be approximated by

$$
\frac{4 \pi|Z|}{Z_{0}} \simeq \frac{1}{\lambda \gamma_{z}^{2}} \simeq \frac{1}{\sigma_{\perp} \gamma_{z}} .
$$

Here and below we ignore numerical factors on the order of unity, so that our formulas are only crude estimates. Our goal is to obtain simple scaling relations helping in orientation in the parameter space.

Note that the impedance is rather flat around the optimal wavelength; it reduces by $10 \%-15 \%$ when the wavelength is by a factor of 2 smaller or larger than the optimal one. The optimal $R_{56}$ of the dispersion section is

$$
R_{56} \simeq \lambda \frac{\gamma}{\sigma_{\gamma}} .
$$

Substituting (9) and (10) into (1), we get an estimate of the amplitude gain per cascade for the wavelength given by (8)

$$
G_{n} \simeq \frac{I}{\sigma_{\gamma} I_{\mathrm{A}}} \frac{L_{d}}{\lambda \gamma_{z}^{2}} .
$$

Thus, the gain per cascade is approximately equal to the longitudinal brightness of the electron beam multiplied by a number of LSC formation lengths.

Let us now consider the limitations on the drift length (5) . The first limit (6) can be rewritten with the help of (9) as

$$
L_{1} \simeq \lambda_{p} \simeq \lambda \gamma_{z}^{2} \sqrt{\frac{\gamma I_{\mathrm{A}}}{I}} .
$$

So, in the case $L_{1}<L_{2}$ the drift space length can be chosen to be $L_{d} \simeq L_{1}$. In this case the expression for the gain (11) reduces to

$$
G_{n} \simeq \frac{1}{\sigma_{\gamma}} \sqrt{\frac{\gamma I}{I_{\mathrm{A}}}} .
$$

This estimate for the gain was originally obtained in [1], it gives the maximal possible gain per cascade of an LSCA. In the considered limit we have a relatively large beta function. It is advisable to reduce it (if technically possible), since the wavelength (8) and length of the drift space (12) are also reduced but the gain (13) stays the same. This happens until the spread of longitudinal velocities starts playing a role, i.e. when $L_{1} \simeq L_{2}$. The corresponding beta function is

$$
\beta_{\mathrm{cr}} \simeq \epsilon \gamma_{z}^{2} \sqrt{\frac{\gamma I_{\mathrm{A}}}{I}} .
$$

If one further reduces beta function, $\beta<\beta_{\mathrm{cr}}$, the maximal drift length is given by $L_{d} \simeq L_{2}$. In this case from (7), (8), and (11) we find that the gain is proportional to the beam brightness in $6 \mathrm{D}$ phase space:

$$
G_{n} \simeq \frac{I}{\sigma_{\gamma} I_{\mathrm{A}}} \frac{\beta}{\epsilon \gamma_{z}^{2}} \simeq \frac{I}{\sigma_{\gamma} I_{\mathrm{A}}}\left(\frac{\lambda}{\epsilon}\right)^{2} .
$$

Although the gain can still be high for $\lambda \gg \epsilon$, it quickly decreases when one goes to shorter wavelengths - contrary to the case (13). Thus, the condition $L_{d} \simeq L_{1} \simeq L_{2}$ (and $\beta \simeq \beta_{\text {cr }}$ ) allows one to get the highest gain at the shortest wavelength. At this point we have

$$
\begin{gathered}
\lambda \simeq \epsilon\left(\frac{\gamma I_{\mathrm{A}}}{I}\right)^{1 / 4}, \\
L_{d} \simeq \epsilon \gamma_{z}^{2}\left(\frac{\gamma I_{\mathrm{A}}}{I}\right)^{3 / 4} .
\end{gathered}
$$

The amplitude gain per cascade is given by (13), the beta function by (14), and the $R_{56}$ by (10).

To estimate the total gain (and number of cascades) required to reach saturation in LSCA, one has to estimate typical density modulation for the start-up from shot noise. The power spectral gain of the amplifier depends on the number of cascades $n$. For an optimal wavelength (8) as a central wavelength (neglecting the dependence of the impedance on $k$ ), and for the optimized $R_{56}$ from (10), one easily obtains from (1) that the total power gain is proportional to $\hat{k}^{2 n} \exp \left(-n \hat{k}^{2}\right)$, where $\hat{k}=k / k_{\text {opt }}$. Thus, the relative bandwidth of the amplifier is in the range 50\%-100\%, depending on the number of cascades. Then an effective shot noise density modulation can be estimated as [11]

$$
\rho_{\mathrm{sh}} \simeq \frac{1}{\sqrt{N_{\lambda}}},
$$

where $N_{\lambda}=I \lambda /(e c)$ is a number of electrons per central wavelength of the amplified spectrum, $\lambda=2 \pi \lambda$. At saturation the density modulation $\rho_{\text {sat }}$ is on the order of unity, so that the total amplitude gain $\rho_{\text {sat }} / \rho_{\text {sh }}$ is

$$
G_{\mathrm{tot}}=G_{1} G_{2} \ldots G_{n} \simeq \sqrt{N_{\lambda}},
$$

where $G_{n}$ is the gain in the $n$th cascade. The total power gain of the saturated amplifier (that shows an enhancement of power in a radiator with respect to spontaneous emission) is simply

$$
G_{\mathrm{tot}}^{(p)}=G_{\mathrm{tot}}^{2} \simeq N_{\lambda} .
$$

We have presented a simple scheme for optimization of LSCA, but we should note that it is not strict and serves for orientation in the parameter space only. For example, one can choose a drift length that is significantly shorter than the limit given by (5) and increase the number of cascades instead. In this case the formula (11) should be used to calculate the gain. For instance, three cascades with the gain 10 in each give about the same total gain as two cascades with the gain 30 in each, but the total length of 
the amplifier (for the same beta function) can be almost twice shorter in the former case. ${ }^{4}$ If in addition one reduces beta function (since the drift space got shorter), one can go to shorter wavelength and higher gain per cascade. So, if the gain per cascade at $\beta \simeq \beta_{\text {cr }}$ is very large, it could be beneficial to go to the limit $L_{d} \simeq L_{2}<L_{1}$-if the beta function is not getting too low for technical realization. On the other hand, in many cases even beta function given by (14) is too small and technically not feasible. In that case, one would have to use larger values of $\beta$ and go to the limit defined by plasma oscillations only, thus using Eqs. (8)-(13). If the wavelength of interest differs significantly from the one given by (8), one should use more general formulas of the previous section. In any case, the formulas of this section are only estimates, and for more accurate gain calculations one should use more general formulas of the previous section (but then one has to specify distributions), and in addition to include dynamics in drifts and chicanes more accurately.

\section{Wavelength compression}

As one can see from the formulas of the optimized LSCA, a typical operating wavelength of an optimized LSCA is significantly longer than a wavelength that can be reached in SASE FELs (they can lase at $\lambda \simeq \epsilon$ ). In order to go to shorter wavelengths for given electron beam parameters in LSCA, one would have to use wavelength compression. The broadband nature of the amplifier makes this option especially attractive. Indeed, the compression factor is given by the formula

$$
C=\left(1-h R_{56}\right)^{-1},
$$

where $h$ is the linear energy chirp (the derivative of relative energy deviation). For a large $C$ a variation of the compression factor reads

$$
\frac{\Delta C}{C} \simeq C \frac{\Delta h}{h}
$$

After the compression the bands of density modulations and of the radiator must overlap. This leads to the following requirement on the compression stability:

$$
\frac{\Delta C}{C}<\frac{\Delta k_{\max }}{k},
$$

where $\Delta k_{\max }=\max \left(\Delta k_{\mathrm{den}}, \Delta k_{\mathrm{rad}}\right)$, and $\Delta k_{\mathrm{den}}$ and $\Delta k_{\mathrm{rad}}$ are bandwidths of the density modulation and of the radiator, respectively. Thus, the stability of the chirp must satisfy the requirement

\footnotetext{
${ }^{4}$ If one assumes that gain is the same in all cascades, gain per cascade is proportional to its length, and the length of a drift is always much larger than the length of a chicane, then from Eq. (19) one gets formally that the shortest total length is achieved at the number of cascades $n \simeq \ln G_{\text {tot }}$, and the gain per cascade $G_{n} \simeq e \simeq 2.718 \ldots$. However, in practice it is advisable to keep gain per cascade at least in the range 5-10.
}

$$
\frac{\Delta h}{h}<\frac{1}{C} \frac{\Delta k_{\max }}{k}
$$

For coherent FEL-type modulations and an undulator as a radiator $\Delta k_{\max } / k \ll 1$ which might set very tight tolerance for the chirp stability and limit practically achievable compression factors. For an LSCA, however, $\Delta k_{\max } / k=$ $\Delta k_{\mathrm{den}} / k \simeq 1$, so that for a given chirp stability one can go for much larger compression. Alternatively, for a given compression factor one can significantly loosen the tolerances. Note also that nonlinearities of the longitudinal phase space do not play a significant role in the case of LSCA.

If the wavelength compression is applied, one should use formula (1) to calculate gain per cascade, and adjust $R_{56}$ to optimize the gain depending on compression factor $C$. One can consider different options for compression. One possibility is to create an energy chirp before the beam enters LSCA. In this case, one can adjust $R_{56}$ in different cascades in order to have a mild compression in each cascade-but this might shift the wavelength beyond the optimal range in the drifts of last cascades. In that case, one should make sure that the number of cascades and their parameters are adjusted such that a saturation of LSCA is finally achieved. Another possibility is to create an energy chirp before the chicane of the last cascade (for instance, by a laser in a short undulator [15-19]), and get the desired compression in the last chicane. In this case, perhaps, one would need very strong energy chirp. An interesting option for a short electron bunch with a high current would be to use an energy chirp, induced by LSC along the whole bunch (then for compression one should use, for instance, doglegs instead of chicanes, taking into account the sign of the energy chirp). In this case, one should carefully adjust parameters of the amplifier cascades since LSC (and a chirp) might strongly increase from one cascade to the next one. However, loose tolerances (24) can make such an option feasible.

\section{E. Undulator}

At the entrance of the undulator we have a chaotically modulated electron beam with a typical amplitude of the order of unity at saturation. The temporal correlations have the scale of a wavelength, and the spectrum is broad. The undulator radiation within the central cone $\sqrt{\lambda / L_{w}}$ (here $L_{w}$ is the undulator length) has a relative bandwidth $N_{w}^{-1} \ll 1$, where $N_{w}$ is the number of undulator periods. In the case when Fresnel number is small, $N_{f}=$ $\sigma_{\perp}^{2} /\left(\lambda L_{w}\right) \ll 1$, the radiation power within the central cone is equal to the power of spontaneous emission (about 0.011 photons per electron are radiated within the central cone of a planar undulator with a large undulator

\footnotetext{
${ }^{5}$ There is, in general, also emission of powerful radiation beyond the central cone.
} 
parameter $K$ ) multiplied by the power gain $N_{\lambda}$ of the LSCA at saturation:

$$
W \simeq W_{\mathrm{sp}} N_{\lambda} .
$$

In this limit the power within the central cone does not depend on the number of undulator periods. One can easily see that the Fresnel number is always small if the condition (8) is satisfied, transverse size of the beam in the undulator is the same as that in amplification cascades, and there is no wavelength compression. In this case the transverse coherence is guaranteed. However, with a strong wavelength compression in the last chicane of LSCA, the Fresnel number might no longer be small, so that one should use a more general formula. For Gaussian transverse distribution of the beam density, and under assumption that the amplified density modulations are transversely correlated, we can use the following expression for the power:

$$
W \simeq W_{\mathrm{sp}} N_{\lambda} F\left(N_{f}\right)
$$

where the suppression factor is [20]

$$
F\left(N_{f}\right)=\frac{2}{\pi}\left[\arctan \left(\frac{1}{2 N_{f}}\right)+N_{f} \ln \left(\frac{4 N_{f}^{2}}{4 N_{f}^{2}+1}\right)\right] .
$$

For a large Fresnel number the last expression reduces to $F\left(N_{f}\right) \simeq 1 /\left(2 \pi N_{f}\right)$, and the power (26) is emitted within the angle $\lambda / \sigma_{\perp}$ which is much smaller than the central cone. In that case we still assume a relatively good transverse coherence if in the drifts of LSCA the wavelength is given by the condition (8) or it is longer. Indeed, transverse correlations are established in this case due to LSC [4]. Longitudinal coherence length, as in the case of spontaneous undulator radiation, is given by the product of the wavelength and the number of undulator periods. We do not discuss in this paper nonlinear harmonic generation in LSCA, since it would be highly speculative without numerical simulations. We can only mention here that this should be possible in a saturated LSCA.

\section{F. Numerical example}

Let us illustrate the formulas of this section with a numerical example. We consider the electron beam with the following parameters: the energy $3 \mathrm{GeV}\left(\gamma \simeq 6 \times 10^{3}\right)$, peak current $2 \mathrm{kA}$, normalized emittance $2 \mu \mathrm{m}$, and rms energy spread $0.3 \mathrm{MeV}\left(\sigma_{\gamma}=0.6\right)$. The energy modulations due to LSC are accumulated in the focusing channels, so that $\gamma_{z}=\gamma$ (no undulators are placed there). From the formula (14) we find $\beta_{\mathrm{cr}} \simeq 1.4 \mathrm{~m}$. Although this low value assumes that the focusing channels are densely packed with the quadrupoles, it is technically possible. From (16) or (8) we get $\lambda \simeq 2.5 \mathrm{~nm}$, i.e., the wavelength $\lambda$ is about $15 \mathrm{~nm}$. The optimal $R_{56}$ for this wavelength is about $25 \mu \mathrm{m}$ (and the chicane may fit in the space between two quadrupoles, so that periodicity of the channel is not disturbed). According to (17), the length of the drift space is about $20 \mathrm{~m}$. The gain per cascade can be found from (13); it is larger than 40. According to (19), the total gain should be about $10^{3}$, so that only two cascades are sufficient. As it was mentioned before, such a high gain per cascade is not optimal from the point of view of the total length of the system, and 3-4 cascades would be more preferable. For instance, for 3 cascades one needs the gain per cascade about 10 , so that the length of a cascade would be about $5 \mathrm{~m}$. The chicanes can be made very compact. Behind the last chicane a tunable-gap undulator with the period length of $5 \mathrm{~cm}$ and a number of periods 30 can be installed. The total length of this system is less than $20 \mathrm{~m}$. The undulator selects a relatively narrow band of about 3\% from the broadband density modulations. The peak power within the central cone is estimated at a gigawatt level, assuming that amplification of density modulation reached saturation in the last cascade. As it was discussed before, a shift of the central wavelength by a factor of 2 does not change the impedance significantly. The gain is also affected weakly as soon as the $R_{56}$ is tuned correspondingly. The undulator wavelength is adjusted by tuning the gap. Therefore, wavelength tunability in the range $7-30 \mathrm{~nm}$ is easily possible with a slightly larger total length of the system.

This numerical example illustrates that LSCA cannot directly compete with FELs in terms of shorter wavelengths (although the wavelength compression can help as one can see from numerical example in Sec. IIIB), higher power and brilliance etc. For instance, a SASE FEL with the given electron beam parameters could successfully saturate at 1-2 nm wavelength within $30-50 \mathrm{~m}$ of undulator length and produce a few gigawatts of peak power within a bandwidth that is smaller than a percent. However, LSCA can be a cheap solution for generation of longer wavelength radiation with a relatively high beam energy, and can have other attractive applications that are discussed in Sec. III.

\section{G. Coherent synchrotron radiation effects in chicanes}

In the above presented considerations, the influence of coherent synchrotron radiation (CSR) inside dispersive elements (chicanes, doglegs, etc.) on the amplification process, as well as on a possible beam degradation, was neglected. Note that the former effect would have been useful, i.e., it could have lead to a higher gain than that estimated in this paper. On the other hand, at the final amplification stage, when the beam is strongly modulated, CSR can be harmful, leading to the emittance growth.

CSR instability in bunch compressor chicanes was studied in detail in [11-13]. It was shown, in particular, that transverse effects (connected with a finite beam emittance) may significantly suppress the instability due to the coupling between transverse and longitudinal phase spaces, described by linear transfer matrix elements $R_{51}$ and $R_{52}$. To estimate a typical magnitude of these elements, one can use simple relations: $R_{51} \simeq \theta$ (a bending angle of 
the chicane) and $R_{52} \simeq d$ (transverse displacement of the beam in the chicane). Beam density modulations are essentially smeared, and CSR effects are strongly suppressed when $R_{51} \sigma_{\perp} / \lambda \gg 1$, and/or $R_{52} \sigma_{\theta} / \lambda \gg 1$. Of course, $R_{51}$ and $R_{52}$ change along the chicane length (they are equal to zero at the beginning and at the end of the chicane), and the suppression is not necessarily effective through the whole chicane. However, if the above mentioned parameters are very big, this usually means that CSR effects are not significant. Quantitatively one can judge this from linear theory $[12,13]$ or from numerical simulations.

There is another effect, leading to a suppression of CSR in chicanes, namely, a transverse beam size (assume that there is no smearing of density modulations). This happens if the following condition is satisfied: $\sigma_{\perp}\left(\lambda^{2} R\right)^{-1 / 3} \gg 1$, where $R$ is the bending radius in a dipole of the chicane.

Typically, all the mentioned transverse effects are large in the case of a short-wavelength LSCA, proposed in this paper. For illustration, let us consider the above presented numerical example of LSCA. Let us assume that the chicane consists of three dipoles $(10 \mathrm{~cm}, 20 \mathrm{~cm}, 10 \mathrm{~cm})$ with the distance between dipoles $5 \mathrm{~cm}$. For the required $R_{56}$, one needs to have a bending angle in the first (last) dipole about 0.01 , i.e. $R \simeq 10 \mathrm{~m}$. Then we estimate $R_{51} \sigma_{\perp} / \lambda \simeq$ 100 , and $R_{52} \sigma_{\theta} / \lambda \simeq 10$, so that one can expect sure smearing of the density modulation inside the chicane. Only in very short parts (beginning of the first dipole and the ending of the last dipole) with the length on the order of $1 \mathrm{~mm}$ the modulations are present, but these parts are much shorter than the CSR formation length, which is of the order of $1 \mathrm{~cm}$. In addition, $\sigma_{\perp}\left(\lambda^{2} R\right)^{-1 / 3} \simeq 5$, i.e., CSR is expected to be strongly suppressed.

\section{POSSIBLE APPLICATIONS OF LSCA}

\section{A. LSCA as a cheap addition to existing or planned $x$-ray FELs}

Undulator beam lines of the existing and planned x-ray FELs often consist of long drift spaces and long undulators. Insertion of a few chicanes and a short undulator at the end may allow for a parasitic production of relatively long wavelength radiation (as compared with the FEL wavelength) by the same electron bunch. This would extend in an inexpensive way the wavelength range of a facility. Moreover, since both radiation pulses are perfectly synchronized, they can be used in pump-probe experiments.

As a first example, let us consider the undulator beam line SASE1 at the European XFEL [21]. There is a long drift space (about $300 \mathrm{~m}$ ) in front of the SASE1 undulator, and $200 \mathrm{~m}$ long drift behind the undulator. The undulator itself has the total length of $200 \mathrm{~m}$ (magnetic length $165 \mathrm{~m}$ plus 35 meters of intersections). Let us consider the electron beam with the following parameters: energy $17.5 \mathrm{GeV}$, normalized slice emittance $0.4 \mathrm{~mm}$ mrad, peak current $3-$ $4 \mathrm{kA}$, and slice energy spread $1.5 \mathrm{MeV}$. The tunable-gap undulator is assumed to be tuned to the resonance with the wavelength $0.05 \mathrm{~nm}$, so that $\gamma_{z}=1.9 \times 10^{4}$. The optimal (for the FEL operation) beta function in the undulator for these beam parameters is about $15 \mathrm{~m}$, and it is about $30-40 \mathrm{~m}$ in the drifts. The core of the bunch with high current saturates at the FEL wavelength in the undulator, so that this part of the bunch is spoiled (has a large energy spread). We consider parts of the bunch with the current about $1 \mathrm{kA}$ assuming that there is no FEL saturation there. We propose to install three compact chicanes: just in front of the undulator, just behind it, and at the end of the second drift. Thus, we have three amplification cascades of LSCA that operate parasitically. The last chicane is followed by a short undulator. From the formulas of the previous section we find that the optimal wavelength for LSC instability is $\lambda \simeq 4 \mathrm{~nm}$. The optimal $R_{56}$ is about $8 \mu \mathrm{m}$ for all cascades. The beta function in all cascades is much larger than $\beta_{\mathrm{cr}}$; moreover, the lengths of all cascades are shorter than reduced wavelength of plasma oscillations, i.e. $L_{d}<L_{1}$. Therefore, we use formula (11) to calculate gain in every cascade. We find that the total gain is given by the following product of partial gains: $G_{\mathrm{tot}} \simeq 8 \times 13 \times 5 \simeq 500$. This is larger than the gain required to reach saturation, about 300 according to (19). We choose an undulator with 50 periods and a period length $10 \mathrm{~cm}$. Radiation power within the central cone exceeds that of spontaneous emission by 5 orders of magnitude and is at sub-GW level with the bandwidth about $2 \%$; radiation is transversely coherent. The tunability can be easily achieved in the range of $2-10 \mathrm{~nm}$ by changing the $R_{56}$ and the undulator gap. The soft $\mathrm{x}$-ray pulses are synchronized with hard $\mathrm{x}$-ray pulses produced by the core of the same bunch, so that these two colors can be used in pump-probe experiments. Alternatively, they can be separated and used by different experiments. ${ }^{6}$

Note that in this example we considered a parasitic use of the beam line and of an unspoiled part of the electron bunch. With a dedicated use of the high-current part of the bunch, one can essentially reduce the total length of the amplifier. Let us consider the same electron bunch as before, but now we assume that the core of the bunch with the current $3 \mathrm{kA}$ is not spoiled by FEL interaction (for instance, some bunches are kicked in front of the undulator by the fast kicker [22]). We consider an operation of LSCA in the drift behind the undulator, requiring beta function to be about $10 \mathrm{~m}$ (somewhat larger that $\beta_{\mathrm{cr}}$ ), thus the optimal wavelength is $2 \mathrm{~nm}$. Choosing length of the drift in an amplification cascade to be $30 \mathrm{~m}$ (it is much smaller than $\lambda_{p}$ ), and the $R_{56} \simeq 4 \mu \mathrm{m}$, we find with the help of (11) that the gain per cascade is about 5 . To reach

\footnotetext{
${ }^{6}$ As an option one can consider the bending system (with properly adjusted $R_{56}$ ) between SASE1 and the downstream soft X-ray undulator SASE3 [21] as an alternative to the last chicane. Then the short undulator is placed in the SASE3 beam line thus extending its wavelength range.
} 
saturation, one would need four cascades, so that the total length of the amplifier would be about $120 \mathrm{~m}$. A gigawattlevel radiation power would then be produced within the central cone of a short undulator; tunability between 1 and $5 \mathrm{~nm}$ is easy to obtain.

Parasitic use of long drifts and unspoiled parts of an electron beam is possible at other facilities, for example, at the soft x-ray FEL user facility FLASH $[23,24]$. There is about $45 \mathrm{~m}$ long drift space in front of the $27 \mathrm{~m}$ long undulator. Without going into the details, we notice that by installing two chicanes (in front of the undulator and behind it, $R_{56} \simeq 200 \mu \mathrm{m}$ ) and a short radiator undulator, one can parasitically generate powerful VUV radiation with the wavelength around $100 \mathrm{~nm}$.

\section{B. Generation of attosecond pulses}

There are many proposals to produce attosecond pulses from FELs $[15-19,25,26]$. In principle, by using strongly nonlinear manipulations with the longitudinal phase space, one can reduce $\mathrm{x}$-ray pulse duration down to several cycles [19]. Here we note that the broadband nature of the LSC instability suggests that few-cycle pulses can be naturally produced in LSCA.

As an example, let us consider the electron beam with the following parameters: the energy $1.5 \mathrm{GeV}$, bunch charge $200 \mathrm{pC}$, peak current $1 \mathrm{kA}$, normalized emittance $0.5 \mu \mathrm{m}$, and rms energy spread $100 \mathrm{keV}\left(\sigma_{\gamma}=0.2\right)$. We choose beta function to be $1.5 \mathrm{~m}$, i.e., much larger than the value one gets from (14). From (16) we get $\lambda \simeq 5 \mathrm{~nm}$, i.e., the wavelength $\lambda$ is about $30 \mathrm{~nm}$. The optimal $R_{56}$ for this wavelength is about $75 \mu \mathrm{m}$, the chicane can be as short as $50 \mathrm{~cm}$. We choose the length of a drift space $L_{d}=1.5 \mathrm{~m}$ which is much smaller than that given by the formula (17). One can find from (13) that the amplitude gain per cascade is about 10, i.e., we need three cascades to reach saturation according to (19).

Now let us introduce wavelength compression into the scheme. This can be done in a way similar to that considered in [19]. In front of the last chicane we install a twoperiod undulator (period length $10 \mathrm{~cm}$ ) which is resonant with the wavelength of $750 \mathrm{~nm}$ of a laser producing pulses with energy of $1 \mathrm{~mJ}$ and duration of $5 \mathrm{fs}$ (FWHM). The maximum of energy modulation can be about $15 \mathrm{MeV}$ and should be adjusted for a desired compression. Note that one can in-couple the laser beam at the position of the previous chicane where the electron beam deviates from the axis by a few millimeters. Consider as an example the compression of a short slice, having the maximal energy slope, by a factor of 7.5, i.e., from 30 to $4 \mathrm{~nm}$. According to (1), the optimal $R_{56}$ for the last chicane is by the compression factor smaller than that of the previous chicanes, i.e., it is $10 \mu \mathrm{m}$ (note that the undulator has a non-negligible $R_{56}=$ $3 \mu \mathrm{m}$ which has to be taken into account). In this case the gain per cascade is the same, i.e., it is about 10 in our example for the compressed slice, what is sufficient for saturation. The current in this slice has increased by a factor of 7.5, and the width of the generated current spike is about $100 \mathrm{~nm}$ (FWHM).

The radiator undulator has 5-10 periods with the period length of $3 \mathrm{~cm}$. The radiated power is estimated with the help of formula (26) at sub-gigawatt level with the pulse duration about 100 attosecond. The contrast is expected to be high since there is no significant amplification in the uncompressed part of the bunch at $4 \mathrm{~nm}$, and saturation at $30 \mathrm{~nm}$ is not achieved (thus there is no harmonics generation) because there is almost no gain in the last cascade due to reduced $R_{56}$. Pulse energy of spontaneous radiation from the whole bunch within coherent angle is at the level of a few percent of the attosecond pulse energy. Note that in this specific example the compression factor could be made larger if a stronger energy modulation by the laser could be achieved. One should also notice that total length of the system is rather small, less than $10 \mathrm{~m}$.

\section{LSCA as a source of radiation with a relatively large bandwidth}

FEL radiation has a narrow band, typically $0.1 \%-1 \%$. For some experiments, however, a relatively large bandwidth is required, up to $10 \%$. In FELs an increase can be achieved by imposing on the electron beam an energy chirp, which is translated into radiation frequency chirp. This approach has technical limits: the accelerator has a finite energy acceptance, and it is not always possible to impose required energy chirps on very short bunches. In an LSCA the density modulation is broadband, and the radiation bandwidth (within the central cone of undulator radiation) is given by an inverse number of undulator periods.

\section{LSCA driven by a laser-plasma accelerator}

The technology of laser-plasma accelerators progresses well [27], a GeV beam is already obtained [28]. The electron beam with the energy about $200 \mathrm{MeV}$ was sent through the undulator, and spontaneous undulator radiation at $18 \mathrm{~nm}$ wavelength was observed [29]. The VUV and $\mathrm{x}$-ray FELs driven by these accelerators are proposed [30,31]. However, it is not clear at the moment if tight requirements on electron beam parameters and their stability, overall accuracy of the system performance, etc., could be achieved in the next years.

Contrary to FELs, the amplification mechanism of LSCA is very robust. For example, it can survive large energy chirps. In the case of an FEL the energy chirp parameter is $\lambda h / \rho^{2}$, where $\rho$ is the FEL parameter [32] defining, in particular, SASE FEL bandwidth. The energy chirp parameter should be small as compared to unity in order to not affect FEL gain. Contrary to that, the mechanism of LSCA is broadband, so that "effective $\rho$ " is on the order of 1 . In other words, in a drift space the influence of the chirp can be always neglected. Of course, if one would like to avoid compression (decompression) in chicanes of 
LSCA, one should require $h R_{56} \ll 1$. If the $R_{56}$ is chosen according to (10), then the condition for the chirp can be formulated as $h \lambda \ll \sigma_{\gamma} / \gamma$.

In an FEL there are stringent requirements on straightness of the trajectory: the electron beam must overlap with radiation over a long distance. In the case of LSCA, one should only require that the angles of the electron orbit be smaller than $\lambda / \sigma_{\perp}$ which means for the optimal wavelength $\gamma_{z}^{-1}$.

One can speculate (since some important parameters of beams have never been measured) that LSCA could be an interesting alternative to FELs, at least as the first step towards building light sources based on laser-plasma accelerators. One of the most important unknown parameters is the slice energy spread (slice size is given by a typical wavelength amplified in LSCA), since the measured value is usually a projected energy spread, dominated by an energy chirp along the bunch. An interesting option would be to use an energy chirp, induced by LSC and wakefields over the whole bunch $[30,33]$, for the wavelength compression as discussed in Sec. II. Taking into account the sign of the energy chirp, one should use, for instance, doglegs instead of chicanes. One can also consider LSCA as a preamplifier (making sure that it does not saturate) with the final amplification in an FEL.

\section{DISCUSSION}

In this paper we introduced the concept of the longitudinal space charge amplifier (LSCA) that can operate in VUV and $\mathrm{x}$-ray ranges. Although such an amplifier cannot directly compete with FELs in terms of shorter wavelength, higher power, brilliance, etc., one can nevertheless find interesting applications for it. In particular, it can be a cheap addition to some existing or planned FEL systems helping to extend the operating range towards longer wavelength and to provide the second color for pump-probe experiments. The broadband nature of the amplifier supports production of short (down to few cycles) VUV and $\mathrm{x}-$ ray pulses. The bandwidth of the radiation from the undulator of LSCA can be controlled by choosing the number of undulator periods. In particular, one can produce powerful radiation with a relatively large bandwidth which might be difficult in an FEL. Robustness of LSCA makes it an interesting alternative to an FEL in light sources driven by laser-plasma accelerators.

There are many different possibilities that were not considered in this paper and are left for future studies. In particular, we did not study nonlinear harmonic generation, an effect that should occur at the saturation of LSCA. Since the amplification mechanism of LSCA is broadband, the bands of harmonics might even partially overlap. The radiation wavelength within the central cone is controlled by tuning the undulator parameter. Also, for a planar undulator there might be a set of odd harmonics on axis.
We have considered in this paper a start-up of LSCA from shot noise. However, LSCA can also amplify a coherently seeded density modulation. In this case, one needs an undulator and a chicane in front of the first cascade of LSCA. The electron beam gets energy modulated by a laser beam in the undulator, and in the chicane these energy modulations are converted into coherent density modulations. Particularly interesting might be a seeding in a fewperiod undulator by attosecond VUV pulses, obtained by high harmonics generation in gases by powerful few-cycle lasers $[34,35]$. Short few-cycle density modulations can be amplified through LSCA without lengthening, and the fewperiod radiator undulator would produce powerful fewcycle VUV radiation. This option is not available in an FEL amplifier due to a narrow bandwidth.

It was briefly mentioned in the paper that LSCA can serve as a preamplifier for a SASE FEL. There might be other options, for instance putting LSCA (with a short undulator and a chicane in front of LSCA) in an optical cavity, thus having, for instance, a regenerative amplifier with a desirable bandwidth. We should also notice here a possibility of using LSCA for some amplification (not necessarily to saturation) of shot noise in light sources based on spontaneous radiation in undulators, for example, in those driven by energy-recovery linacs. In this way, one can significantly enhance radiation intensity and brilliance.

Finally, we have to mention that a possibility of a harmful LSC instability at short wavelengths (VUV and soft $\mathrm{x}$ ray) should not be forgotten. Such an instability can develop parasitically in FEL systems (at wavelengths that are much longer than the FEL wavelength) with dispersive elements, such as chicanes in high-gain harmonic generation schemes (especially dangerous can be "fresh bunch" chicanes), achromatic bends for separation of beam lines, chicanes in seeding and self-seeding schemes, etc. If LSC instability develops to a significant level of density modulations, strong energy modulations (acting as local energy spread) can be induced in the last parts of FELs thus hampering their operation.

\section{ACKNOWLEDGMENTS}

We would like to thank R. Brinkmann, M. Dohlus, P. Emma, F. Gruener, Z. Huang, A. Mesek, and M. Venturini for useful discussions.

[1] E. L. Saldin, E. A. Schneidmiller, and M. V. Yurkov, Nucl. Instrum. Methods Phys. Res., Sect. A 483, 516 (2002).

[2] E. L. Saldin, E. A. Schneidmiller, and M. V. Yurkov, Nucl. Instrum. Methods Phys. Res., Sect. A 528, 355 (2004).

[3] Z. Huang et al., Phys. Rev. ST Accel. Beams 7, 074401 (2004).

[4] M. Venturini, Phys. Rev. ST Accel. Beams 11, 034401 (2008). 
[5] D. F. Ratner, A. Chao, and Z. Huang, in Proceedings of the 30th FEL Conference, Gyeongju, Korea, 2008, p. 338 [http://www.jacow.org].

[6] H. Loos et al., in Proceedings of the 30th FEL Conference, Gyeongju, Korea, 2008, p. 485 [http://www.jacow.org].

[7] S. Wesch et al., in Proceedings of the 31st FEL Conference, Liverpool, 2009, p. 619 [http://www.jacow.org].

[8] A. Lumpkin et al., Phys. Rev. ST Accel. Beams 12, 080702 (2009).

[9] Z. Huang et al., Phys. Rev. ST Accel. Beams 13, 020703 (2010).

[10] A. M. Kondratenko and E. L. Saldin, Part. Accel. 10, 207 (1980).

[11] E. L. Saldin, E. A. Schneidmiller, and M. V. Yurkov, Nucl. Instrum. Methods Phys. Res., Sect. A 490, 1 (2002).

[12] S. Heifets, G. Stupakov, and S. Krinsky, Phys. Rev. ST Accel. Beams 5, 064401 (2002).

[13] Z. Huang and K.-J. Kim, Phys. Rev. ST Accel. Beams 5, 074401 (2002).

[14] G. Geloni et al., Nucl. Instrum. Methods Phys. Res., Sect. A 578, 34 (2007).

[15] E. L. Saldin, E. A. Schneidmiller, and M. V. Yurkov, Opt. Commun. 237, 153 (2004).

[16] A. A. Zholents and W. M. Fawley, Phys. Rev. Lett. 92, 224801 (2004).

[17] A. A. Zholents and G. Penn, Phys. Rev. ST Accel. Beams 8, 050704 (2005).

[18] E. L. Saldin, E. A. Schneidmiller, and M. V. Yurkov, Phys. Rev. ST Accel. Beams 9, 050702 (2006).
[19] D. Xiang, Z. Huang, and G. Stupakov, Phys. Rev. ST Accel. Beams 12, 060701 (2009).

[20] E. L. Saldin, E. A. Schneidmiller, and M. V. Yurkov, Nucl. Instrum. Methods Phys. Res., Sect. A 539, 499 (2005).

[21] M. Altarelli et al., DESY Report No. 2006-097, DESY, Hamburg, 2006 (see also http://xfel.desy.de).

[22] R. Brinkmann, E. A. Schneidmiller, and M. V. Yurkov, Nucl. Instrum. Methods Phys. Res., Sect. A 616, 81 (2010).

[23] W. Ackermann et al., Nat. Photon. 1, 336 (2007).

[24] K. Tiedtke et al., New J. Phys. 11, 023029 (2009).

[25] E. L. Saldin, E. A. Schneidmiller, and M. V. Yurkov, Opt. Commun. 212, 377 (2002).

[26] P. Emma, Z. Huang, and M. Borland, in Proceedings of the 26th FEL Conference, Trieste, Italy, 2004, p. 333 [http:// www.jacow.org].

[27] E. Esarey, C. B. Schroeder, and W. P. Leemans, Rev. Mod. Phys. 81, 1229 (2009).

[28] W. P. Leemans et al., Nature Phys. 2, 696 (2006).

[29] M. Fuchs et al., Nature Phys. 5, 826 (2009).

[30] F. Gruener et al., Appl. Phys. B 86, 431 (2007).

[31] C. B. Schroeder et al., in Proceedings of the 28th FEL Conference, Berlin, 2006, p. 455 [http://www.jacow.org].

[32] R. Bonifacio, C. Pellegrini, and L. M. Narducci, Opt. Commun. 50, 373 (1984).

[33] G. Geloni et al., Nucl. Instrum. Methods Phys. Res., Sect. A 578, 34 (2007).

[34] R. Kienberger et al., Nature (London) 427, 817 (2004).

[35] G. Sansone, Science 314, 443 (2006). 